\title{
Prognostic factors of patients with Gliomas - an analysis on 335 patients with Glioblastoma and other forms of Gliomas
}

\author{
Jianfeng Liang ${ }^{1}$, Xiaomin Lv², Changyu Lu', Xun Ye ${ }^{1,3}$, Xiaolin Chen ${ }^{1,3}$, Jia Fu', Chenghua Luo ${ }^{4^{*}}$ (D) and
} Yuanli Zhao ${ }^{1,3^{*}}$

\begin{abstract}
Background: The prognosis of glioma is poor, despite recent advances in diagnosis and treatment of the disease. It is important to investigate the clinical characteristics and prognostic factors of glioma so as to provide basis for treatment and management of patients.

Method: A total of 335 patients with glioma were included in this study. These patients were admitted to the medical center between November 2015 and December 2018. The clinical data, including demographic data, tumor characteristics, treatment strategy, expression pattern of tumor markers, and survival data, were retrospectively reviewed. Survival data were analyzed using Kaplan-Meier curves with log-rank test, while multivariate analysis Cox regression model was used to investigate risk factors for mortality.

Results: In this patient cohort, glioblastoma (40\%), diffuse glioma (14.6\%) and oligodendroglioma (9.6\%) were the most common pathological types. The expression of Ki-67 was associated with several clinicopathological parameters (e.g. tumor type, grade, and number of lesions). In addition, Ki-67 correlated with the mortality within the first year of the post-treatment follow-up $(P<0.001)$. Kaplan-Maier analysis revealed that older patients $(\geq 45$ years) displayed worse prognosis than those aged under 45 years $(P=0.038)$. Dismal prognosis was also associated with clinical parameters, including high tumor grade, multiple lesions, and Karnofsky performance score (KPS). Multivariate analysis showed that low KPS $(<85)$ increased the risk of mortality by 2.3 folds with a $95 \% \mathrm{Cl}$ of 1.141 to $4.776(P=0.020)$. Low tumor grade (grade $1-2)$ oppositely reduced the mortality risk by 0.22 folds $(95 \% \mathrm{Cl}, 0.065$ to $0.763, P=0.0168)$.
\end{abstract}

Conclusion: KPS and tumor grade were independent prognostic factors in patients with gliomas.

Keywords: Gliomas, Glioblastoma, Clinical characteristics, Survival, Prognostic factors

\section{Background}

There are more than 100,000 cases of central nervous system (CNS) cancer diagnosed each year worldwide [1], and gliomas represent $40 \%$ of all brain tumors [2]. There are different types of gliomas, namely astrocytoma, oligodendroglioma, glioblastoma (GBM), and diffuse glioma. Among which, GBM is the most common brain

\footnotetext{
* Correspondence: luochenghua@pkuih.edu.cn; zhaoyuanli@pkuih.edu.cn ${ }^{4}$ Department of Retroperitoneal Tumors Surgery, Peking University International Hospital, No.1 Science Park Road, ZGC Life Science Park, Beijing 102206, China

'Department of Neurosurgery, Peking University International Hospital, No.1 Science Park Road, ZGC Life Science Park, Beijing 102206, China Full list of author information is available at the end of the article
}

neoplasms [3]. Studies showed that the disease was slightly male predominance (male to female ratio of approximately 1.4:1) $[4,5]$. The clinical presentation of a patient with GBM can vary greatly depending on the stage and location of the tumor; some symptoms include headache, seizure, and progressive neurologic deficits, in which seizure is a symptom observed in as many as $25 \%$ of patients [6,7].

Current standard therapy for gliomas includes maximal safe surgical resection, radiation, and chemotherapy with temozolomide [8]. Although there have been recent advances in diagnosis and treatment of the malignancy, the prognosis of gliomas is still poor, especially for those

(c) The Author(s). 2020 Open Access This article is distributed under the terms of the Creative Commons Attribution 4.0 International License (http://creativecommons.org/licenses/by/4.0/), which permits unrestricted use, distribution, and reproduction in any medium, provided you give appropriate credit to the original author(s) and the source, provide a link to the Creative Commons license, and indicate if changes were made. The Creative Commons Public Domain Dedication waiver (http://creativecommons.org/publicdomain/zero/1.0/) applies to the data made available in this article, unless otherwise stated. 
patients with malignant and invasive gliomas. The highly invasive nature prevents complete resection of the tumor, causing significant neurologic morbidity and mortality. For example, the median survival of patients with GBM was only 15 months and a median progression-free survival was 6.2 to 7.5 months $[3,5]$. To improve the quality of life and survival time of patients, it is important to investigate prognostic factors of the disease, so the high risk patient group can be identified and treated with an aggressive regime. Some clinical-pathological parameters have been proposed. For example, Ki-67 was found as a reliable indicator of tumor cell proliferation [9], but its value in predicting prognosis remains controversial.

In this study, we investigated clinical manifestations and prognostic factors of patients with gliomas through a retrospective analysis of clinical characteristics and follow-up data of 335 patients. The data might provide insights for improving the treatment and management of patients with different types of gliomas.

\section{Patients and methods}

\section{Study population}

The present study was a retrospective study on $335 \mathrm{pa}-$ tients with gliomas. These patients were admitted to our hospital for medical treatment between November 2015 and December 2018. Eligible patients included those with their gliomas pathologically confirmed. Patients were excluded if they had other brain tumors or systemic diseases. The study was reviewed and approved by the Ethics Committee and Institutional Review Board of our hospital. Informed consent was obtained from each of the participants.

\section{Data collection}

Clinical data were retrieved for statistical analysis from patient's medical records. The data included patient's demographic data, tumor characteristics (i.e. lesion sites, pathological classification, grade, etc.), treatment approaches (i.e. surgical resection, radiotherapy, and chemotherapy), tumor markers expression (i.e. Ki-67, GFAP, p53, etc.), and survival time after treatment.

\section{Statistical analysis}

Retrieved data were analyzed using statistical software SAS version 9.3. Normally distributed continuous variables were analyzed using Student's t-test, with the ones not normally distributed be examined using Wilcoxon two sample test. Survival data were studied using Kaplan-Meier curve with log-rank test, with the differences between survival curves evaluated using TukeyKramer corrected $P$ values. Risk factors for mortality after treatment were identified by multivariate analysis using Cox regression model. Significant differences were indicated by $P$ value $<0.05$.

\section{Results}

Baseline characteristics of the enrolled patients

A total of 335 patients with glioblastoma were enrolled in this study (Table 1). There were 190 males and 145 females, with their ages ranging from 1 to 86 years. Epilepsy was complained in 97 patients (29\%). Glioblastoma (40\%),

Table 1 Demographic data, tumor characteristics and treatment strategies of patients

\begin{tabular}{|c|c|c|}
\hline \multicolumn{2}{|c|}{ Clinicopathological parameters } & \multirow{2}{*}{$\begin{array}{l}\text { Frequency(\%) } \\
190(56.72)\end{array}$} \\
\hline Gender & Male & \\
\hline & Female & $145(43.28)$ \\
\hline \multirow[t]{2}{*}{ Epilepsy } & No & $238(71.04)$ \\
\hline & Yes & $97(28.96)$ \\
\hline \multirow[t]{8}{*}{ Location of lesion } & Parietal and/or junction & $35(10.45)$ \\
\hline & Forehead & $89(26.57)$ \\
\hline & Fronto-temporal & $18(5.37)$ \\
\hline & Brainstem & $12(3.58)$ \\
\hline & Cerebellum & $10(2.99)$ \\
\hline & Right temporal & $77(22.99)$ \\
\hline & Left temporal & $21(6.27)$ \\
\hline & Other & $73(21.79)$ \\
\hline \multirow[t]{8}{*}{ Pathological type } & Malignant astrocytoma & $21(6.27)$ \\
\hline & Malignant oligodendroglioma & $16(4.78)$ \\
\hline & Glioblastoma & $134(40.00)$ \\
\hline & Diffuse glioma & 49 (14.63) \\
\hline & Oligodendroglioma & $32(9.55)$ \\
\hline & Astrocytoma & $23(6.87)$ \\
\hline & Other & $23(6.87)$ \\
\hline & Unknown & $37(11.04)$ \\
\hline \multirow[t]{4}{*}{ Tumor grade } & 1 & $10(3.21)$ \\
\hline & 2 & $87(27.88)$ \\
\hline & 3 & $60(19.23)$ \\
\hline & 4 & $155(49.68)$ \\
\hline \multirow[t]{2}{*}{ Lesion number } & Single & $303(90.45)$ \\
\hline & Multiple & $32(9.55)$ \\
\hline \multirow[t]{2}{*}{ Surgical treatment } & No & $56(16.72)$ \\
\hline & Yes & $279(83.28)$ \\
\hline \multirow[t]{3}{*}{ Surgical approach } & Subtotal resection & $67(24.01)$ \\
\hline & Gross resection & $205(73.48)$ \\
\hline & Unknown & $7(2.51)$ \\
\hline \multirow[t]{3}{*}{ Radiotherapy } & No & $51(15.22)$ \\
\hline & Yes & $115(34.33)$ \\
\hline & Unknown & $169(50.45)$ \\
\hline \multirow[t]{3}{*}{ Chemotherapy } & No & $52(15.52)$ \\
\hline & Yes & 116 (34.63) \\
\hline & Unknown & 167 (49.85) \\
\hline
\end{tabular}


diffuse glioma (14.6\%) and oligodendroglioma (9.6\%) were the three most common pathological types, and for tumor grading, $46.7 \%$ of the cases were classified as grade 4 .

Most patients (83.3\%) were treated by surgery, of which subtotal resection and gross total resection constituted 24 and $73.5 \%$ of the cases, respectively. These surgical approaches were associated with neither patients' survival outcomes nor any clinicopathological parameters. About one-third of the patients received radiotherapy (34.3\%) and chemotherapy (34.6\%). Survival outcomes after treatment were followed up. Up to the time point when data were retrieved for analysis, the survival and mortality rate was 51.8 and $20 \%$, respectively (i.e. $28.2 \%$ of the cases were not followed).

\section{Clinical correlation of Ki-67 level}

The associations of Ki-67 with clinicopathological parameters were studied in 232 of 335 enrolled patients, who had clinical record of Ki-67 level (Table 2). Ki-67 level was associated with patients' age $(P<0.001)$. The level also correlated the occurrence of epilepsy $(P=0.007)$. For pathological parameters, Ki-67 was associated with tumor type $(P<0.001)$, grade $(P<0.001)$, and number of lesions (single vs multiple) $(P=$ 0.023). Ki-67 also correlated with Karnofsky performance score (KPS; $P=0.017$ ) and several markers like neuronal nuclei (NeuN; $P=0.003$ ), epithelial membrane antigen (EMA; $P<0.001)$, p53 $(P<0.001)$ and NF $(P=0.023)$.

Ki-67 was found not significantly associated with whether and how the surgery was done. Notably, Ki-67 correlated strongly with the mortality within the first year of the post-treatment follow-up $(P<0.001)$. In addition, patients with elevated Ki-67 (i.e. $\geq 20$ ) showed shorter survival than those with low Ki-67 (i.e. <20) within a post-treatment period of 40 months $(P=0.002)$ (Fig. 1).

\section{Clinicopathological parameters associated with the first- year survival}

The factors affecting the first year survival of patients after treatment were examined (Table 3). Univariate analysis

Table 2 Correlation of Ki-67 with clinicopathological parameters in gliomas

\begin{tabular}{|c|c|c|c|c|}
\hline \multicolumn{2}{|c|}{ Clinicopathological parameters } & \multicolumn{2}{|l|}{ Ki-67 level } & \multirow[t]{2}{*}{$P$ value } \\
\hline & & Low $(<20)$ & High ( $\geq 20)$ & \\
\hline \multirow[t]{2}{*}{ Age } & $<45$ & 77 & 35 & $<0.001$ \\
\hline & $\geq 45$ & 39 & 81 & \\
\hline \multirow[t]{2}{*}{ Epilepsy } & No & 70 & 89 & 0.007 \\
\hline & Yes & 46 & 27 & \\
\hline \multirow[t]{7}{*}{ Pathological type } & Malignant astrocytoma & 9 & 4 & $<0.001$ \\
\hline & Malignant oligodendroglioma & 2 & 11 & \\
\hline & Glioblastoma & 16 & 82 & \\
\hline & Diffuse glioma & 30 & 6 & \\
\hline & Oligodendroglioma & 25 & 0 & \\
\hline & Astrocytoma & 16 & 1 & \\
\hline & Other & 18 & 12 & \\
\hline \multirow[t]{2}{*}{ Tumor grade } & $1-2$ & 71 & 1 & $<0.001$ \\
\hline & $3-4$ & 42 & 113 & \\
\hline \multirow[t]{2}{*}{ Lesion number } & Single & 112 & 103 & 0.023 \\
\hline & Multiple & 4 & 13 & \\
\hline \multirow[t]{2}{*}{ KPS } & $<85$ & 58 & 76 & 0.017 \\
\hline & $\geq 85$ & 58 & 40 & \\
\hline \multirow[t]{2}{*}{ NeuN } & Positive & 21 & 7 & 0.003 \\
\hline & Negative & 86 & 106 & \\
\hline \multirow[t]{2}{*}{ EMA } & Positive & 7 & 32 & $<0.001$ \\
\hline & Negative & 95 & 76 & \\
\hline \multirow[t]{2}{*}{ P53 } & Positive & 58 & 97 & $<0.001$ \\
\hline & Negative & 38 & 12 & \\
\hline \multirow[t]{2}{*}{$N F$} & Positive & 49 & 43 & 0.023 \\
\hline & Negative & 10 & 23 & \\
\hline
\end{tabular}


suggested that tumor pathological type and grade were only marginally associated $(P=0.05)$. The first-year survival also correlated with KPS $(P<0.001)$ and different markers including Ki-67 $(P=0.004)$, and Syn $(P=0.048)$. Multivariate analysis using Cox regression model was also performed to identify the risk factor of mortality within the first year posttreatment. It was shown that low KPS (i.e. $<85)$ was the only factor that substantially elevated the risk (OR, 5.965; 95\% CI, 1.996 to $17.822 ; P=0.001$ ).

\section{Clinicopathological parameters associated with the overall survival}

Kaplan-Maier analysis with log rank test revealed multiple clinicopathological parameters associated with patients' overall survival (Fig. 2). Older patients (i.e. $\geq 45$ years) displayed worse prognosis than those aged under 45 years $(P=0.038)$. Patients with no epilepsy also showed a poorer prognosis compared to their counterparts with epilepsy $(P=0.027)$. Dismal prognosis was also associated with certain tumor pathology like high tumor grade (i.e. grade $3-4)(P<0.001)$ and multiple lesions $(P=0.026)$. Patients with KPS $<85$ also presented less favorable survival outcomes compared to those with KPS $\geq 85(P=0.001)$.

Low KPS (i.e. $<85$ ) was identified by multivariate analysis as a factor to increase the risk of mortality by 2.3 folds with a 95\% CI of 1.141 to $4.776(P=0.020)$. Low tumor grade (i.e. grade $1-2$ ) oppositely reduced the mortality risk by 0.22 folds ( $95 \% \mathrm{CI}, 0.065$ to $0.763, P=$ 0.0168).

\section{Discussion}

Surgical resection and postoperative radiotherapy and chemotherapy can significantly delay tumor progression, however, the recurrence rate is unacceptably high, making
Table 3 Clinicopathological parameters associated with the first-year survival after treatment

\begin{tabular}{lllll}
\hline Clinicopathological parameters & Survival & Mortality & $P$ value \\
\hline KPS & $<85$ & 24 & 26 & $<0.001$ \\
& $\geq 85$ & 45 & 6 & \\
Ki-67 & $<20$ & 26 & 5 & 0.004 \\
& $\geq 20$ & 21 & 20 & \\
Syn & Positive & 33 & 17 & 0.048 \\
& Negative & 9 & 0 & \\
\hline
\end{tabular}

the overall cure rate of gliomas low. With a hope to improve the treatment outcome of patients, there has long been a search for clinical parameters of prognostic values in the clinical decision of treatment strategy. Early studies have suggested many factors affecting the prognosis of glioma patients, however, most have remained elusive in their effects $[10,11]$. Other clinical parameters like tumor grade, age of onset, surgical approaches, and the use of postoperative adjuvant therapy are also shown to be associated with the prognosis of patients $[12,13]$. Over the last decade, with the advances in genomic and proteomic profiling, many molecular markers have emerged as prognostic indicators for glioma. A four-microRNA signature was shown able to identify patients with lower-grade gliomas under high risk of mortality [14]. A low serum level of microRNA-376 was identified as an independent factor predicting poor outcome of glioma patients [15]. A mutation of BRAF, V600E, was associated with an improved overall survival among glioma patients [16]. However, despite the usefulness of these molecular markers in predicting survival in their respective defined single cohorts, the prognostic power of these markers across multiple patient populations has yet to be further validated. It has remained important to examine the clinical parameters associated with the treatment outcome of glioma patients.

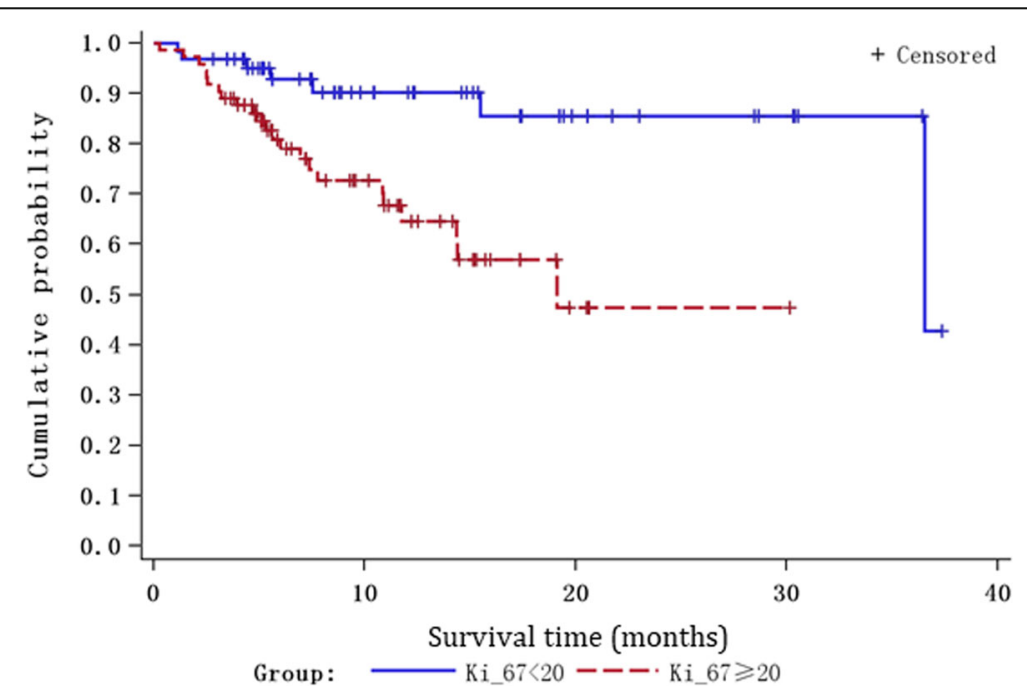

Fig. 1 Kaplan-Meier analysis showing the significant difference in survival between glioma patients with low and high Ki-67 expression 

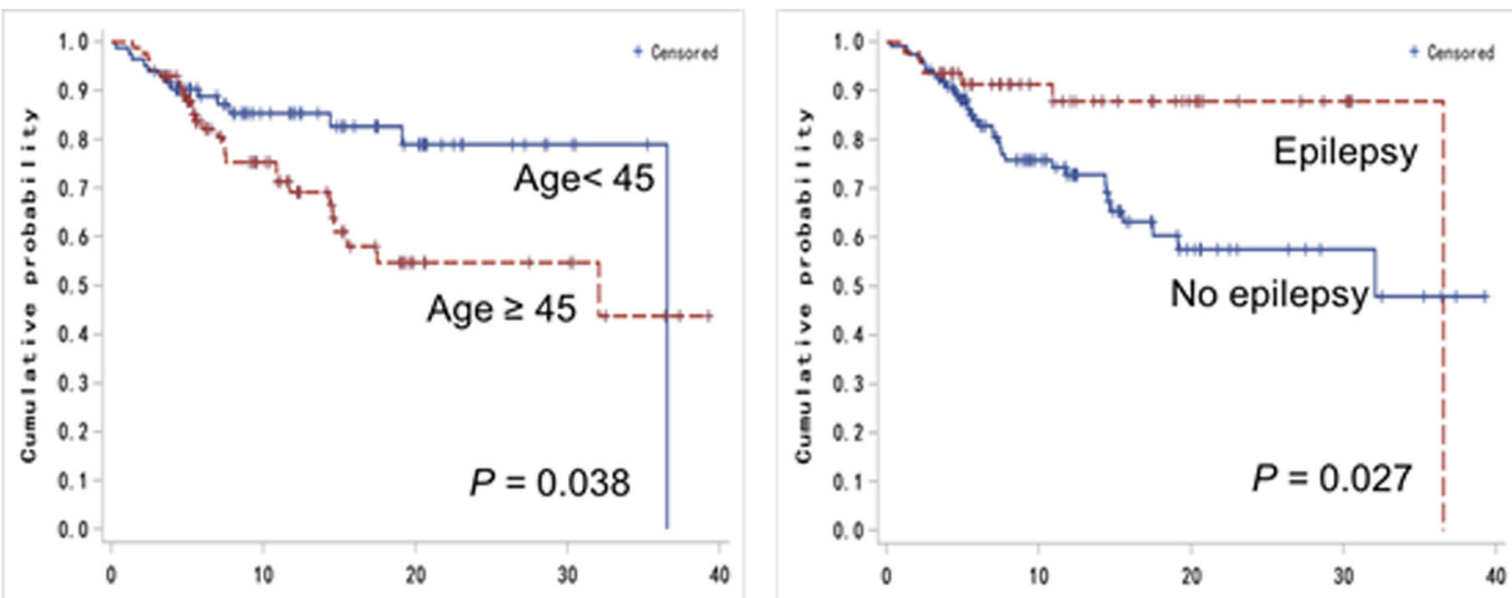

Survival time (months)

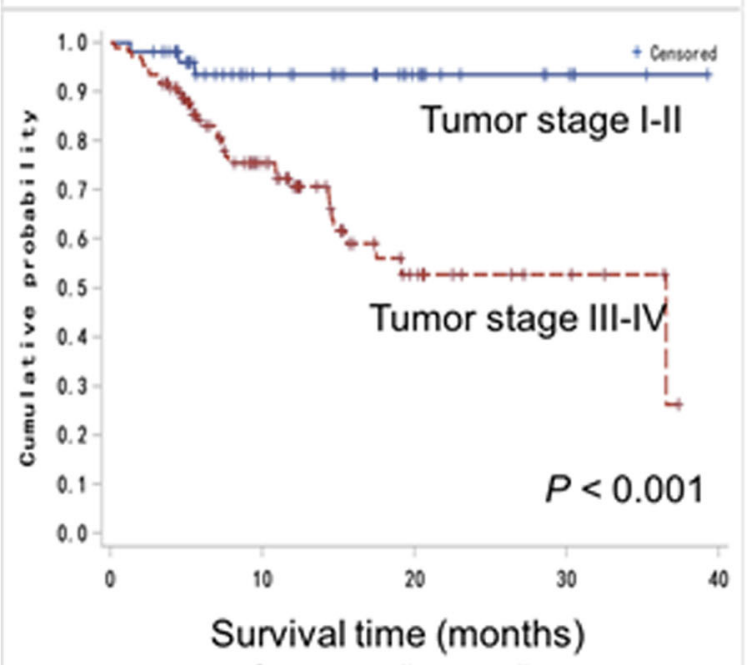

Survival time (months)
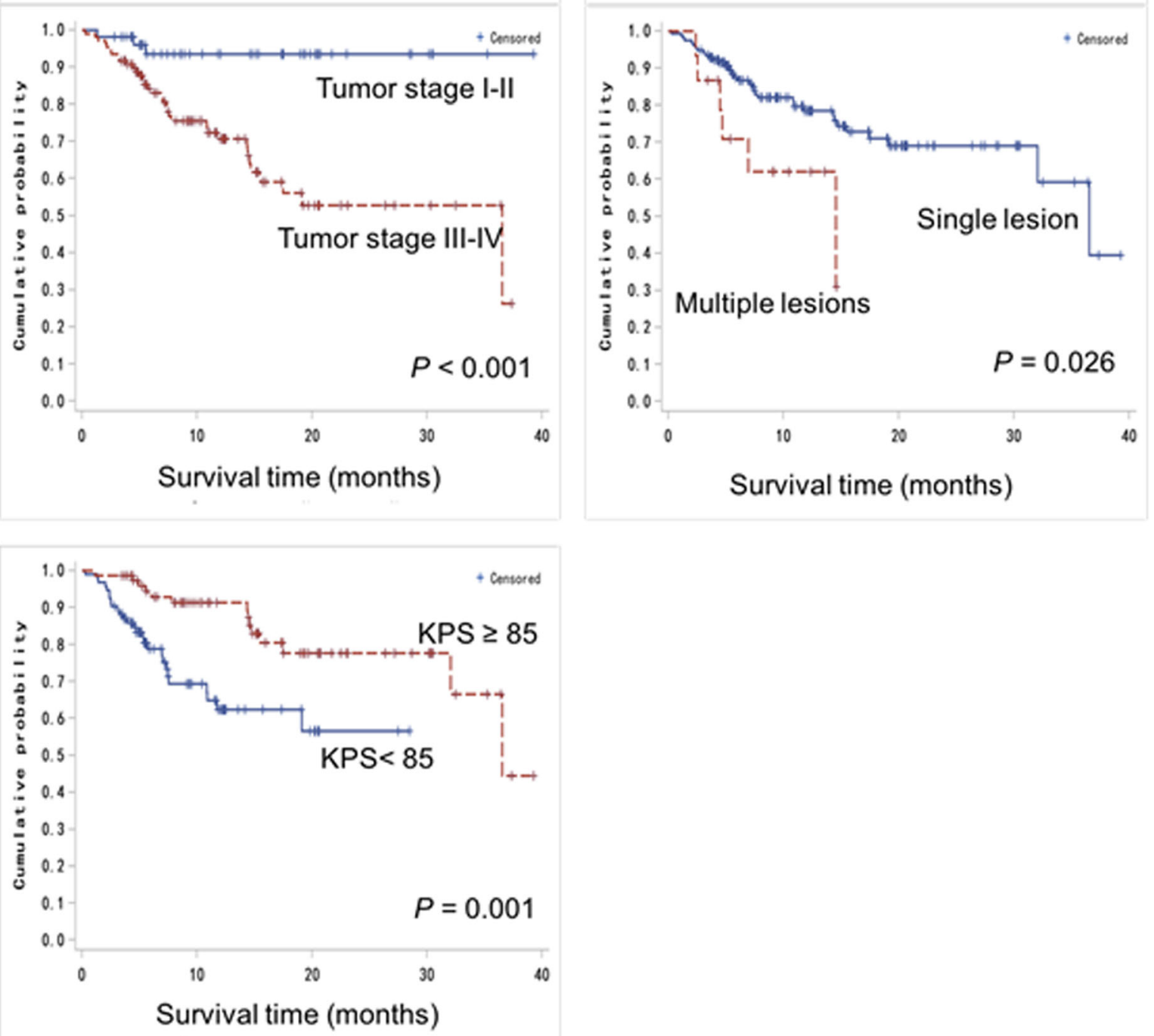

Fig. 2 Significant association of clinical parameters with the overall survival of glioma patients as examined by Kaplan-Meier analysis 
The present study examined the clinical associations of different clinicopathological parameters in 335 patients with glioma. Our analysis clearly suggested that old age, high tumor grade, multiple lesions, and low KPS are associated with the poor survival of the patients. Multivariate analysis further indicated that low KPS and low tumor grade can significantly elevate and reduce, respectively, the risk of mortality of patients. These findings are clinically relevant. The cohort size of the present study is relatively large when compared to those of the published, covering patients with different types of gliomas and those treated with different therapies. In addition, among the different types of gliomas found in our cohort, $40 \%$ of them were glioblastoma. This resembles most clinical situations in which glioblastoma is the most common types of gliomas worldwide.

Our analysis suggested that low KPS is an independent risk factor for mortality within the first year after treatment and in long-term survival of glioma patients. This finding is in line with many studies showing KPS is a promising prognostic indicator in patients with glioma. For example, patients with KPS $>70$ were shown to survive longer than their counterparts with KPS $<70$ [17]. The combined use of KPS with MGMT promoter methylation and patient age in a recursive partitioning analysis modal can also accurately predict the prognosis of patients with glioblastomas [18]. Our analysis also identified low tumor grade as a predictor of good survival. Indeed, tumor grades and KPS score are known clinical parameters already in practice. However, the present study defined a cutoff value of KPS that would be useful in Chinese patients with gliomas. Whether the combined use of KPS and tumor grade would further improve the prediction has yet to be fully examined.

\section{Conclusion}

In WHO 2016 classification of brain tumors, molecular sub-grading has been established and is presently in routine clinical use. To provide more useful insights into the refinement of management strategy for patients with gliomas, the present study was aimed identify several clinical parameters associated with the prognosis of patients. The findings nevertheless require further validation in independent cohorts of glioma patients. In our study cohort, although patients received different treatments like radiotherapy, chemotherapy or combined treatment, the association of these treatments with patient's prognosis has remained to be established. The prognostic value of clinical parameters combined with molecular markers has also yet to be examined. Furthermore, in vitro and in vivo experiments will be done to depict the clinical significance of certain molecular parameters in glioma.
Abbreviations

GBM: Oligodendroglioma, glioblastoma; KPS: Karnofsky performance score

\section{Acknowledgements}

The authors wish to acknowledge the help received from all the members in Department of Neurosurgery, Peking University International Hospital.

\section{Authors' contributions}

Conceptualization: $J, X L, C L, J Z$; investigation: $J$, $X L, X Y$; formal analysis: $X C, J F$; resources: $C L, Y Z$; writing and original draft preparation: $J$, $X L$; supervision: $J F, C L, Y Z$; writing the review and editing: all authors. All authors read and approved the final manuscript.

\section{Funding}

This study was supported by The National Natural Science Foundation of China (No. 81301092) and Peking University International Hospital Research Grant (No. YN2017ZD02). Funding bodies did not play any role in the design of the study, or collection, analysis or interpretation of data, or in writing the manuscript.

\section{Availability of data and materials}

The datasets generated and analyzed during the current study are available from the corresponding author on reasonable request.

\section{Ethics approval and consent to participate}

The study protocol was approved by the Ethics Committee of Peking University International Hospital. Written informed consent was obtained from all the study subjects before enrollment.

\section{Consent for publication}

Not applicable

\section{Competing interests}

The authors declare that they have no competing interests.

\section{Author details}

${ }^{1}$ Department of Neurosurgery, Peking University International Hospital, No.1 Science Park Road, ZGC Life Science Park, Beijing 102206, China.

${ }^{2}$ Department of Neurology, The First Hospital of Jilin University, Changchun, 130021 Jilin Province, China. ${ }^{3}$ Department of Neurosurgery, Beijing Tiantan Hospital, Capital Medical University, Beijing 100050, China. ${ }^{4}$ Department of Retroperitoneal Tumors Surgery, Peking University International Hospital, No.1 Science Park Road, ZGC Life Science Park, Beijing 102206, China.

Received: 4 October 2019 Accepted: 30 December 2019

Published online: 15 January 2020

References

1. Parkin DM. Global cancer statistics in the year 2000. Lancet Oncol. 2001;2: 533-43.

2. Turner JD, Williamson R, Almefty KK, et al. The many roles of microRNAs in brain tumor biology. Neurosurg Focus. 2010;28:E3.

3. Thakkar JP, Dolecek TA, Horbinski C, et al. Epidemiologic and molecular prognostic review of glioblastoma. Cancer Epidemiol, Biomark Prev Publication Am Assoc Cancer Res, Cosponsored Am Soc Prev Oncol. 2014; 23:1985-96.

4. Chen S, Tanaka S, Giannini C, et al. Gliomatosis cerebri: clinical characteristics, management, and outcomes. J Neuro-Oncol. 2013;112: 267-75.

5. Stupp R, Taillibert S, Kanner AA, et al. Maintenance therapy with tumortreating fields plus Temozolomide vs Temozolomide alone for Glioblastoma: a randomized clinical trial. JAMA. 2015;314:2535-43.

6. Perry J, Zinman L, Chambers A, et al. The use of prophylactic anticonvulsants in patients with brain tumours-a systematic review. Curr Oncol. 2006;13:222-9.

7. Schiff D, Lee EQ, Nayak $L$, et al. Medical management of brain tumors and the sequelae of treatment. Neuro-oncol. 2015;17:488-504.

8. Minniti G, Muni R, Lanzetta G, et al. Chemotherapy for glioblastoma: current treatment and future perspectives for cytotoxic and targeted agents. Anticancer Res. 2009;29:5171-84. 
9. Hu X, Miao W, Zou Y, et al. Expression of p53, epidermal growth factor receptor, Ki-67 and O (6)-methylguanine-DNA methyltransferase in human gliomas. Oncol Lett. 2013;6:130-4.

10. Sarbu N, Oleaga L, Valduvieco I, et al. Increased signal intensity in FLAIR sequences in the resection cavity can predict progression and progressionfree survival in gliomas. Neurocir. 2016;27:269-76.

11. Danielson PD, Zottoli SJ, Corrodi JG, et al. Localization of choline acetyltransferase to somata of posterior lateral line efferents in the goldfish. Brain Res. 1988;448:158-61.

12. Dahlrot RH. The prognostic value of clinical factors and cancer stem cellrelated markers in gliomas. Dan Med J. 2014;61:B4944.

13. Ampie L, Choy W, Lamano JB, et al. Prognostic factors for recurrence and complications in the surgical management of primary chordoid gliomas: a systematic review of literature. Clin Neurol Neurosurg. 2015;138:129-36.

14. Qian $Z$, Li Y, Fan X, et al. Prognostic value of a microRNA signature as a novel biomarker in patients with lower-grade gliomas. J Neuro-Oncol. 2018; 137:127-37.

15. Huang Q, Wang C, Hou Z, et al. Serum microRNA-376 family as diagnostic and prognostic markers in human gliomas. Cancer Biomark: Sect A Dis Markers. 2017;19:137-44.

16. Vuong HG, Altibi AMA, Duong UNP, et al. BRAF mutation is associated with an improved survival in Glioma-a systematic review and meta-analysis. Mol Neurobiol. 2018;55:3718-24.

17. Bigner DD, Brown MT, Friedman AH, et al. lodine-131-labeled antitenascin monoclonal antibody 81C6 treatment of patients with recurrent malignant gliomas: phase I trial results. J Clin Oncol : Official J Am Soc Clin Oncol. 1998; 16:2202-12

18. Yang $F$, Yang $P$, Zhang $C$, et al. Stratification according to recursive partitioning analysis predicts outcome in newly diagnosed glioblastomas. Oncotarget. 2017;8:42974-82

\section{Publisher's Note}

Springer Nature remains neutral with regard to jurisdictional claims in published maps and institutional affiliations.

Ready to submit your research? Choose BMC and benefit from:

- fast, convenient online submission

- thorough peer review by experienced researchers in your field

- rapid publication on acceptance

- support for research data, including large and complex data types

- gold Open Access which fosters wider collaboration and increased citations

- maximum visibility for your research: over $100 \mathrm{M}$ website views per year

At $\mathrm{BMC}$, research is always in progress.

Learn more biomedcentral.com/submissions 